\title{
4. Einführung in die Online-Benutzerforschung zu Digitalen Bibliotheken
}

\author{
Elke Greifeneder
}

\begin{abstract}
„We were naive enough to think that it would be relatively straightforward to transfer research strategies developed for studying face-to-face contexts to life online“"

Markham und Baym, 2009
\end{abstract}

\section{Einleitung}

Digitale Angebote sind heute elementarer Bestandteil von Bibliotheken, eine benutzerfreundliche Gestaltung derselben ist daher unerlässlich. Aber wie schafft man Angebote, die den Bedürfnissen und Wünschen der Benutzer entsprechen? Trotz einer Vielzahl goldener Regeln ${ }^{1}$, Ratschlägen ${ }^{2}$ und Richtlinien ${ }^{3}$ gibt es nichts, was allgemein für alle Benutzer und alle Angebote zutrifft. Ein benutzerorientiertes digitales Angebot kann nur durch wiederkehrende (iterative) Studien zu und mit Benutzern erreicht werden. ${ }^{4}$

Doch wie untersucht man Benutzer, die zu jeder Zeit und von jedem beliebigen Ort aus die eigenen Angebote nutzen können? Dieser Beitrag stellt Methoden der Online-Benutzerforschung bei digitalen Angeboten vor und weist zugleich auf einige Tücken dieser Methoden hin.

Als Online-Forschung werden Studien bezeichnet, die einen doppelten Bezug zum Internet haben: das Internet ist sowohl ihr Gegenstand als auch das Medium ihrer Methode. Der Forscher erhebt Daten mit dem Internet über das Internet. ${ }^{5}$ Im angloamerikanischen Raum spricht man auch von „Internet Research“6 ${ }^{\text {o- }}$

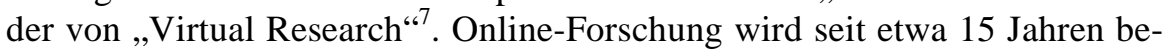
trieben. Bis heute hat sie den Status einer eigenständigen Disziplin nicht erreicht.

\footnotetext{
1 Shneiderman; Plaisant, 2005.

2 Krug; Dubau, 2006.

3 U.a. Nielsen, 2000.

4 Siehe die Kapitel im Methodenteil dieses Bandes.

5 Welker; Wenzel, 2010.

6 Markham; Baym; Nancy, 2009.

7 Buchanan, 2004.
} 
Ein Charakteristikum von Online-Studien ist die räumliche Distanz zwischen Forscher und Teilnehmer; dies wird als „remote“ (engl. für „entfernt“) bezeichnet. Von synchronen Remote-Studien spricht man, wenn - wie beispielsweise bei Chat-Interviews - eine räumliche Distanz, aber keine zeitliche gegeben ist. Bei asynchronen Remote-Studien gibt es zusätzlich eine zeitliche Distanz. Ein Beispiel hierfür wäre eine Online-Umfrage, da Teilnehmer zu jeder Zeit an einem Ort ihrer Wahl daran teilnehmen können.

\section{Grundlagen der Online-Benutzerforschung}

Der größte Reiz digitaler Angebote ist zugleich das größte Hindernis für Studien: das Fehlen jeglicher zeitlicher und räumlicher Einschränkungen. Wie soll man aber Benutzer aus China oder den USA zur Teilnahme an einer Fokusgruppe in Deutschland überzeugen? Wie soll man synchrone Chat-Interviews führen, wenn es in Seattle früher Nachmittag, in Berlin jedoch vier Uhr in der Früh ist?

Der digitale Benutzer ist häufig ein gesichtsloses Wesen, von dem man selten mehr weiß, als dass es aus irgendeinem unbekannten Grund auf das Angebot klickt, dort eine bestimmte Aktion durchführt und dann - zufrieden oder nicht - das Angebot wieder verlässt. Das Problem des gesichtslosen Benutzers ist nicht neu und seit Jahren gibt es eine kaum endende Reihe von Publikationen, die sich mit Recherchegewohnheiten oder Design-Wünschen von Benutzern sowie möglichen zukünftigen Modellen wie einem OPAC 2.0 beschäftigen. Beispielhaft sei hier auf das Beluga-Projekt an der Staats- und Universitätsbibliothek Hamburg ${ }^{8}$, die Ermittlung möglicher Nutzerprofile einer digitalen Buchgeschichte ${ }^{9}$ und auf die dänische Studie „The hybrid library: from the $u$ sers perspective ${ }^{\text {“10 }}$ hingewiesen. Letztere untersuchte Verhaltensmuster von Benutzern, um Persona ${ }^{11}$ digitaler Angebote zu entwickeln.

Eines hat sich bei allen Studien herauskristallisiert: die alten Unterscheidungen mittels demographischer Angaben (wie Alter, Geschlecht, Studiengang etc.) haben nur bedingt Gültigkeit im digitalen Raum. Ein 14-Jähriger hat möglicherweise eine höhere Computerkompetenz als ein 40-Jähriger. Gruppierungen von Benutzern in digitalen Umgebungen besitzen die meiste Aussagekraft, wenn bei der Erhebung nach Erfahrung und Wissen gefragt wird.

Eine zweite Herausforderung für Online-Benutzerstudien ist der Kontext des Benutzers im Umgang mit digitalen Angeboten. Taylor ${ }^{12}$ schrieb bereits lange bevor der Computer Einzug in den Alltag hielt, über das Phänomen ,the

\footnotetext{
8 Beluga, 2011.

9 Greifeneder; Kuhn; Rühr, 2010.

10 Akselbo, 2006.

11 Siehe Kapitel 1 Grundlagen des Usability-Engineerings.

12 Taylor, 1991.
} 
home as an information use environment". Denn nicht nur die Kenntnisse des Benutzers haben Einfluss auf die Interaktion mit digitalen Angeboten. So nutzen manche Benutzer digitale Angebote von ihrem beruflichen Arbeitsplatz aus, haben an diesen Rechnern aber häufig nur eingeschränkte Rechte. Verwendet man Angebote, die eine Installation von Software erfordern, wird der Benutzer automatisch ausgeschlossen. Ein Beispiel ist der virtuelle Rundgang der Universitätsbibliothek Bielefeld, der nur mit dem Internet Explorer und nur nach Installation eines Plugins betretbar ist (Stand Mai 2011). Die Hinzunahme des Benutzungskontextes kann zudem wichtige Hinweise zur Dateninterpretation liefern. So bewerten Menschen Angebote anders, wenn sie schlecht gelaunt oder abgelenkt sind. ${ }^{13}$

Der vorliegende Aufsatz behandelt speziell die Methoden der OnlineBenutzerforschung und kann daher die allgemeinen Grundlagen der Benutzerforschung nicht in der gebotenen Ausführlichkeit darstellen. Ein grundlegendes Verständnis über Methoden der Benutzerforschung vermitteln beispielsweise die folgenden beiden, sehr empfehlenswerten Werke: Der Bericht der Digital Library Federation Usage and Usability Assessment ${ }^{14}$ erläutert in äußerst prägnanter Form die verschiedenen Methoden sowie deren jeweiligen Vor- und Nachteile. Als Grundlagenwerk zur Vorbereitung eigener Studien eignet sich das Buch Observing the User Experience von Kuniavsky ${ }^{15}$. Darin wird die Anwendung verschiedener Methoden detailliert vorgestellt. Beide Werke behandeln die neuen Methoden der Online-Forschung jedoch nur am Rande.

Einige Methoden der Online-Forschung verwenden Werkzeuge („Tools“), die genuin für sie entwickelt wurden (wie der Web-Fragebogen oder die Remote-Usability-Tools); andere benutzen Werkzeuge, die für vielerlei Zwecke Verwendung finden (wie Chaträume oder virtuelle Videokonferenzräume).

Die Methoden werden nach quantitativen und qualitativen Aspekten unterschieden. ${ }^{16}$ Qualitative Forschung erkundet einen noch unbekannten Sachverhalt. Wenn man etwas noch nicht kennt, kann man es nicht messen. Ein Beispiel: Eine Bibliothek will wissen, ob ihre Benutzer vier jüngst eingeführte Angebote kennen und schätzen. Mit einem Fragebogen kann die Bibliothek konkret abfragen, ob Angebot B bekannt ist und ob Angebot C gegenüber Angebot B bevorzugt wird. Will eine Bibliothek jedoch wissen, welche zusätzlichen Angebote im Internet sie künftig anbieten soll, muss sie zuerst herausfinden, welche Angebote von Interesse sein könnten. Man könnte in diesem Fall qualitativ erkunden, welche neuen Angebote gewünscht sind. Qualitative Forschung arbeitet mit sehr kleinen Teilnehmergruppen; Ziel ist eine hohe Inhaltsvalidität und ein tiefer Informationsgehalt der Ergebnisse. Dafür verzichtet qualitative

13 Greifeneder, 2011.

14 Troll Covey, 2002.

15 Kuniavsky, 2003.

16 Vgl. folgend Norlin; Winter, 2000. 
Forschung auf den Anspruch, repräsentative Aussagen zu gewinnen. Sie bejaht bewusst die Subjektivität der Ergebnisse. Beispiele sind Interviews oder Beobachtungen.

Quantitative Forschung hingegen dient zur objektiven Messung und Quantifizierung von Sachverhalten. Ein Forscher muss also bereits wissen, was er messen will. Quantitative Forschung verwendet standardisierte Verfahren und eignet sich zum Testen von Hypothesen und für wiederkehrende Untersuchungen. Für quantitative Studien verwendet man große Stichproben. Beispiele sind Fragebogen oder Logfile-Analysen.

Nicht immer kann man eine Methode ausschließlich einer Kategorie zuordnen: Ein Fragebogen kann sowohl quantitativen als auch qualitativen Charakter haben. Bei der Methode des Lauten Denkens werden sowohl quantitative Daten erhoben (z.B. wie viele Teilnehmer eine Aufgabe in welcher Zeit erfolgreich gelöst haben) als auch qualitative Daten erfasst (wie etwa individuelle Rückmeldungen zu Problemen). Beispiele für qualitative und quantitative Daten gibt Tabelle 1.

Beispiel für qualitative Daten
„Ich benutze das Webangebot eigentlich
immer als erste Anlaufstelle für meine wis-
senschaftliche Arbeit. Neulich habe ich ein
Buch gefunden, dass neu digitalisiert war und über die integrierte Volltextsuche habe ich entdeckt, dass in einer Fußnote zum ersten Mal der Begriff Ausländerfeindlichkeit verwendet wird. Das war für meine Forschung sehr wichtig. Was mich jedoch total nervt, ist die Unübersichtlichkeit der Suche. Ich glaube, dass in diesem Angebot total viele Schätze schlummern, aber ich muss immer erst einmal alle möglichen Tricks anwenden, um etwas zu finden.“ (Teilnehmer Peter Schmidt)

Tabelle 1: Beispiele für qualitative und quantitative Daten
Beispiel für quantitative Daten

95 Prozent der Teilnehmer an der Umfrage gaben an, dass das Webangebot eine wichtige Informationsquelle ist.

56 Prozent benutzen das Angebot mehrmals in der Woche.

Nur 9 Prozent unserer Besucher fanden auf Anhieb, was sie suchten.

\section{Web-Fragebogen}

Web-Fragebogen (umgangssprachlich pauschal als Online-Umfragen bezeichnet) sind seit etwa 2002 die beliebteste Methode in der Online-Forschung, da sie verhältnismäßig leicht in der technischen Umsetzung sind. Fertige Produkt- 
lösungen wie LimeSurvey oder SurveyMonkey ermöglichen es, mit wenig Aufwand Fragen in ein Webformular einzugeben, den fertigen Fragebogen auf einer Website zu verlinken und dank guter Exportfunktionen nicht nur die Rohdaten, sondern eine Aufbereitung derselben in Diagrammen und Charts für Präsentationen herunterzuladen. Die technisch einfache Umsetzung ermöglicht es, kostengünstig und schnell einen Web-Fragebogen zu erstellen.

Doch die technische Einfachheit täuscht über die Schwierigkeiten der Fragenerstellung und Einschränkungen in der Deutung der Daten hinweg. Die richtige und eindeutig formulierte Frage zu stellen, erfordert ein hohes Maß an Problembewusstsein. So dürfen Fragen keine Antworten suggerieren. Wenn man Teilnehmer einer Studie fragt: „Viele Benutzer wünschen sich eine FacebookGruppe ihrer Bibliothek. Soll die Bibliothek Ihrer Meinung nach eine solche Gruppe gründen?", beeinflusst man bereits die Antwort durch den Verweis, dass viele andere Benutzer das auch so sehen. Fragen über die Zukunft sind ebenfalls sehr schwer zu konzipieren: Wenn man Benutzer fragt, ob diese sich vorstellen können, E-Books zu nutzen, dann ist die Qualität der gewonnen Daten sehr dünn. Denn man weiß nicht viel mehr, als dass die Benutzer es sich heute vorstellen oder eben nicht vorstellen können. Dies sagt nichts über den tatsächlichen Bedarf in einigen Jahren aus. Hätte Mark Zuckerberg im Jahr 2000 in einer Studie gefragt, ob die Teilnehmer sich vorstellen könnten, ihr Privatleben in sozialen Netzwerken auszubreiten, dann hätte er Facebook wahrscheinlich nie entwickelt.

Man muss sich bei Fragebogen bewusst machen, dass sie immer nur ein von Benutzern selbst eingeschätztes Verhalten erfassen: das heißt, man fragt einen Benutzer, was er auf einer Website gemacht hat oder welches Angebot er gerne hätte. Dieses Verhalten muss aber nicht mit dem tatsächlichen Verhalten übereinstimmen. Die Gründe dafür sind vielfältig: der Benutzer möchte nicht über den wahren Sachverhalt Auskunft geben, er kann sich nicht mehr genau daran erinnern oder er glaubt tatsächlich, dass er wie angegeben handelte. Wirkliche Sicherheit, dass das vorgegebene Verhalten dem tatsächlichen entspricht, erlangt man mit einem Web-Fragebogen nie. Hier bietet sich eine Methodenkombination an, die parallel das tatsächliche Verhalten misst.

Bei Fragebogen gibt es - grob eingeteilt - vier Formen der Fragestellung: Bei der ersten Form muss der Benutzer sich für eine aus mehreren vorgegebenen Antwortmöglichkeiten entscheiden. Bei der zweiten Form, der Mehrfachauswahl, können mehrere Antwortvorgaben ausgewählt werden. Bei der dritten Form, dem Rating, soll der Benutzer eine Wertung abgeben. Bei der letzten Form kann der Benutzer in ein freies Feld einen Kommentar schreiben. Die ersten drei Formen generieren quantitative Daten, die letzte Form generiert qualitative Daten. Abbildung 1 illustriert die vier Fragetypen.

Nimmt man sich ausreichend Zeit für die Entwicklung der Fragen, ist der Web-Fragebogen eine sehr praktikable Methode für die Online-Forschung. Ein Web-Fragebogen kann problemlos auf einer Website als Link oder Pop-Up 
platziert werden, oder es wird die Einladung zur Teilnahme per E-Mail an einzelne Benutzer oder Mailinglisten verschickt. Es gibt bei Web-Fragebogen aus technischer Sicht weder räumliche noch zeitliche Einschränkungen.

*1 Wie sind Sie momentan mit dem Internet verbunden?

über einen schnellen DSL-Zugang oder eine schnelle ISDN-Verbindung zu Hause

über eine mobile Verbindung wie HSDPA, UMTS, EDGE oder GPRS

über ein Modem

anders

2

Welche Services Ihrer Bibliothek nutzen Sie gegenwärtig?

Ich habe den Newsletter der Bibliothek abonniert

Ich nutze den Online-Katalog (OPAC)

Ich bin ein Freund der Facebook-Gruppe der Bibliothek

$\square$ Ich bekomme Erinnerungen als SMS auf mein Handy

3

Ich stimme den folgenden Aussagen zu (1) oder stimme überhaupt nicht zu (5)

\begin{tabular}{l|c|c|c|c|c|}
\hline & 1 & 2 & 3 & 4 & 5 \\
\hline Die Website wirkt sehr professionell gestaltet & 0 & 0 & 0 & 0 & 0 \\
\hline $\begin{array}{l}\text { Die angezeigten Suchtreffer waren relevant } \\
\text { Die Suche hat sehr gut funktioniert }\end{array}$ & 0 & 0 & 0 & 0 & 0 \\
\hline
\end{tabular}

4

Bitte beschreiben Sie kurz, nach was Sie in der digitalen Bibliothek gesucht haben:

Abb. 1: Vier Varianten der Frageform in Web-Fragebogen

\section{Logfile-Analysen}

Im Gegensatz zu Web-Fragebogen messen Logfile-Analysen das tatsächliche Nutzerverhalten. Logfiles (oder richtigerweise im Deutschen als Logdateien übersetzt) geben Auskunft, an welcher Stelle ein Benutzer wann mit dem Webangebot interagiert. Etymologisch stammt der Ausdruck aus der Schifffahrt, bei der man in Logbüchern aktuelle Schiffspositionen eingetragen hat. Das Aufzeichnen (engl. Tracking) dieser Interaktionsdaten in Logfiles und die gezielte Auswertung derselben nennt man Logfile-Analyse.

Die Methode eignet sich sehr gut, um Muster in der Benutzung zu erkennen: woher kommen die Benutzer, wann sind Stoßzeiten in der Benutzung oder welche Angebote erzeugen überdimensional viele Fehlermeldungen? Viele Studien verwenden Logfile-Analysen, um Aussagen über die Nutzung eines 
Angebots wie Seitenaufrufe, Downloads oder Nulttreffermeldungen zu erhalten. Diese Daten sind rein quantitativer Natur. Verfolgt man gezielt den Weg eines bestimmten Benutzers, spricht man von einer Transaction-Log-Analysis, kurz TLA, oder auch von Click-Stream-Analysis.

Die Schwierigkeiten der Logfile-Analyse bestehen in der richtigen Interpretation der erhaltenen Daten. Was definiert einen Besuch eines OnlineAngebots? Ein Klick auf die Startseite oder Klicks auf jede beliebige Unterseite, über die ein Benutzer durch eine Suchmaschine gelangt sein könnte? Muss ein Besuch eine Mindestdauer haben - zählt ein Benutzer schon, wenn er eine Site nur aufgerufen und sofort wieder verlassen hat? Kann man immer genau zwischen menschlichem Besucher und Suchmaschinenroboter unterscheiden? Was definiert man als eine missglückte Suche? Ist das eine Nulltreffermeldung, oder sind auch zu viele Suchtreffer eine missglückte Suche?

Logfiles haben einen weiteren Nachteil: die Daten sagen nur, welche Interaktionen stattgefunden haben, nicht aber warum. Der Forscher erfährt also nur, dass ein Benutzer immer wieder auf einen bestimmten Link geklickt hat. Die Frage nach den Gründen hierfür kann nicht durch die Logfiles beantwortet werden.

Ähnlich wie der Web-Fragebogen ist die Logfile-Analyse in der technischen Umsetzung eine verhältnismäßig einfach anzuwendende Methode, da die meisten Systeme bereits Logfiles erfassen und man die Daten nur abrufen muss. Logfiles sind ebenso wie die Web-Fragebogen ohne räumliche und zeitliche Einschränkung nutzbar. Sie sind eine unaufdringliche Form der Datensammlung, da der Benutzer in der Regel nicht weiß, dass Daten über ihn erhoben werden. IP-Adressen dürfen aus datenschutzrechtlichen Gründen jedoch nie gesammelt werden.

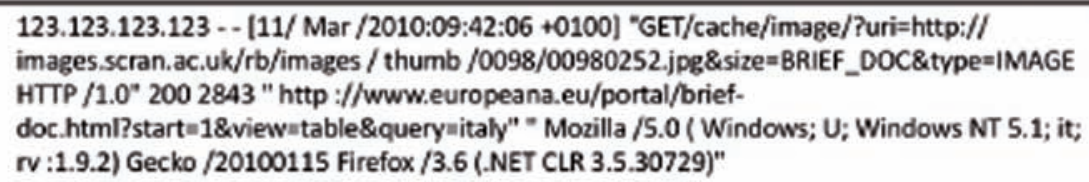

Abb. 2: Beispiel von Logfile-Daten einer digitalen Bibliothek

Viele Datenbankanbieter offerieren die Bereitstellung von fertig ausgewerteten Logfiles. Allerdings basieren die daraus gebildeten Statistiken nicht alle auf den gleichen Algorithmen und gleichen Begriffsdefinitionen. So nützlich diese Daten sind, sie verlieren in der Anwendung mangels Vergleichbarkeit an Wert. Wenn zum Beispiel die Statistiken der Fachverlage Elsevier und Emerald den Besuch einer Website unterschiedlich definieren, dann kann man diese Statistiken nicht miteinander vergleichen. 


\section{Mouse- und Clicktracking}

Zwei andere Methoden nutzen Logfiles, um eine visuelle Analyse der WebsiteBenutzung zu ermöglichen. Diese beiden Verfahren sind das Mousetracking (deutsch: das Verfolgen des Mauszeigers) und das Clicktracking (das Verfolgen von Klicks). Je nach Verfahren wird aufgezeichnet, wie ein Benutzer den Mauszeiger bewegt oder auf welche Stellen oder Links Benutzer klicken. Mit einer guten Mousetracking-Software kann man ähnlich hochwertige Ergebnisse wie mit einem konventionellen Eyetracking-Test erzielen (Eyetracking: das Verfolgen der Blickbewegungen von Probanden). Ziel beider Verfahren ist es herauszufinden, auf welche Bereiche einer Website Benutzer die meiste Aufmerksamkeit richten, damit man wichtige Informationen an diesen Stellen platzieren kann.

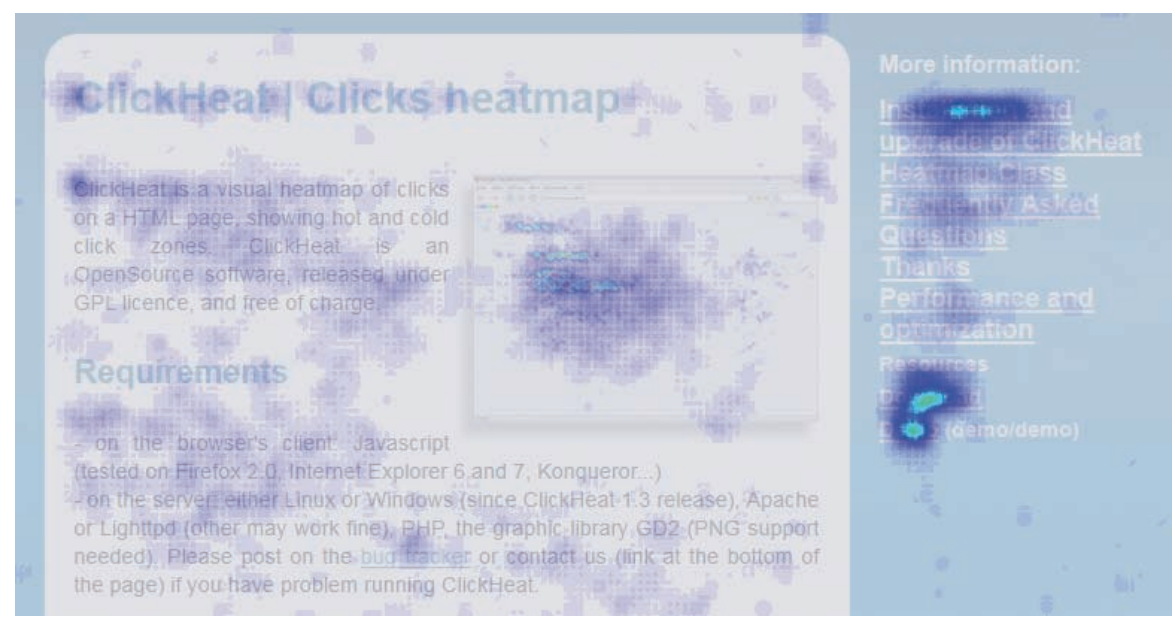

Abb. 3: Visualisierung der Benutzer-Aktivitäten durch Heatmaps (Abbildung der Software ClickHeat)

Häufigste Visualisierungsform von Mouse- und Clicktracking-Verfahren sind sogenannte Heatmaps, welche die Klicks oder Mausbewegungen in Form einer Warm-Kalt-Darstellung präsentieren (Abb. 3). Beide Methoden ermöglichen eine räumlich und zeitlich unabhängige Anwendung und sind somit für die Online-Forschung geeignet. Zur Verwendung der Technik ist die Installation einer Software nötig. 


\section{Remote-Usability-Testing}

Nicht wegzudenken aus der Online-Forschung sind Usability-Studien (deutsch: Studien zur Benutzerfreundlichkeit oder Gebrauchstauglichkeit). Diese Studien untersuchen, inwieweit die Struktur und Gestaltung eines Angebots den Bedürfnissen der Benutzer entspricht. Detaillierte Informationen zum UsabilityEngineering und zu den verschiedenen Methoden zur Umsetzung von Usability finden sich in anderen Kapiteln dieses Buches.

Die meisten Usability-Methoden haben den Nachteil, dass Sie ortsgebunden sind; das heißt, Teilnehmer müssen in der Regel in einem Testlabor vor Ort erscheinen. Doch gerade bei der Untersuchung digitaler Angebote ist es problematisch, wenn man ausschließlich lokal verfügbare Benutzer einbezieht.

Eine gängige Online-Methode zur Evaluation von Usability sind standardisierte Web-Fragebogen, die in den letzten Jahren wieder besonders ,stark in Mode gekommen" sind. ${ }^{17}$ Benutzer werden darin gefragt, von wo sie auf eine Seite zugegriffen haben, wie häufig sie die Seite benutzen, nach was sie gerade recherchiert haben oder welche Probleme bei der Recherche aufgetreten sind. Der Nachteil an dieser Methode ist offensichtlich: Die erhaltenen Daten entsprechen ausschließlich dem Eindruck, die der Benutzer von seinem Verhaltens selbst hat (,claimed behavior"). Wie der Nutzer tatsächlich vorgegangen ist, stimmt häufig nicht exakt mit seiner Selbstauskunft überein. Man erhält dadurch sehr ungenaue Daten.

Seit etwa 2008 haben Remote-Usability-Tests stark an Bedeutung gewonnen. Bei dieser Form des Tests können Benutzer ortsungebunden und zum Teil sogar zeitungebunden an Studien teilnehmen. Bei synchronen RemoteUsability-Tests nutzt man gängige Internet-Tools, um mit den Teilnehmern in Kontakt zu treten. Besonders häufig werden Werkzeuge zur Bildschirmfreigabe (engl. screen sharing) verwendet. Der Teilnehmer erlaubt dabei dem Forscher, Zugriff auf den eigenen Bildschirm zu bekommen, und ruft dann die zu untersuchende Website auf. Über Chat oder besser noch via Internet-Telefonie („Voice over IP“, kurz: VoIP) stellt man Fragen und Aufgaben, die der Teilnehmer der Studie dann auf dem eigenen Rechner ausführt. Das Testdesign ähnelt dem laborbasierten Think-Aloud-Test (Methode des „Lauten Denkens“). Synchrone Remote-Usability-Test sind in diesem Sinn keine neue Methode, sondern nur eine technische Brücke, die es ermöglicht, Labor-Methoden auch Remote durchzuführen.

Die Vorteile synchroner Remote-Usability-Tests sind ihre weltweite Anwendbarkeit und die geringen Kosten. Ein Vorteil gegenüber den asynchronen Tests ist die aktive Interaktion mit dem Teilnehmer, die Rückfragen erlaubt. Nachteilig wirken sich die Abhängigkeit von bestimmten Uhrzeiten aus, die

17 Beschnitt, 2009. 


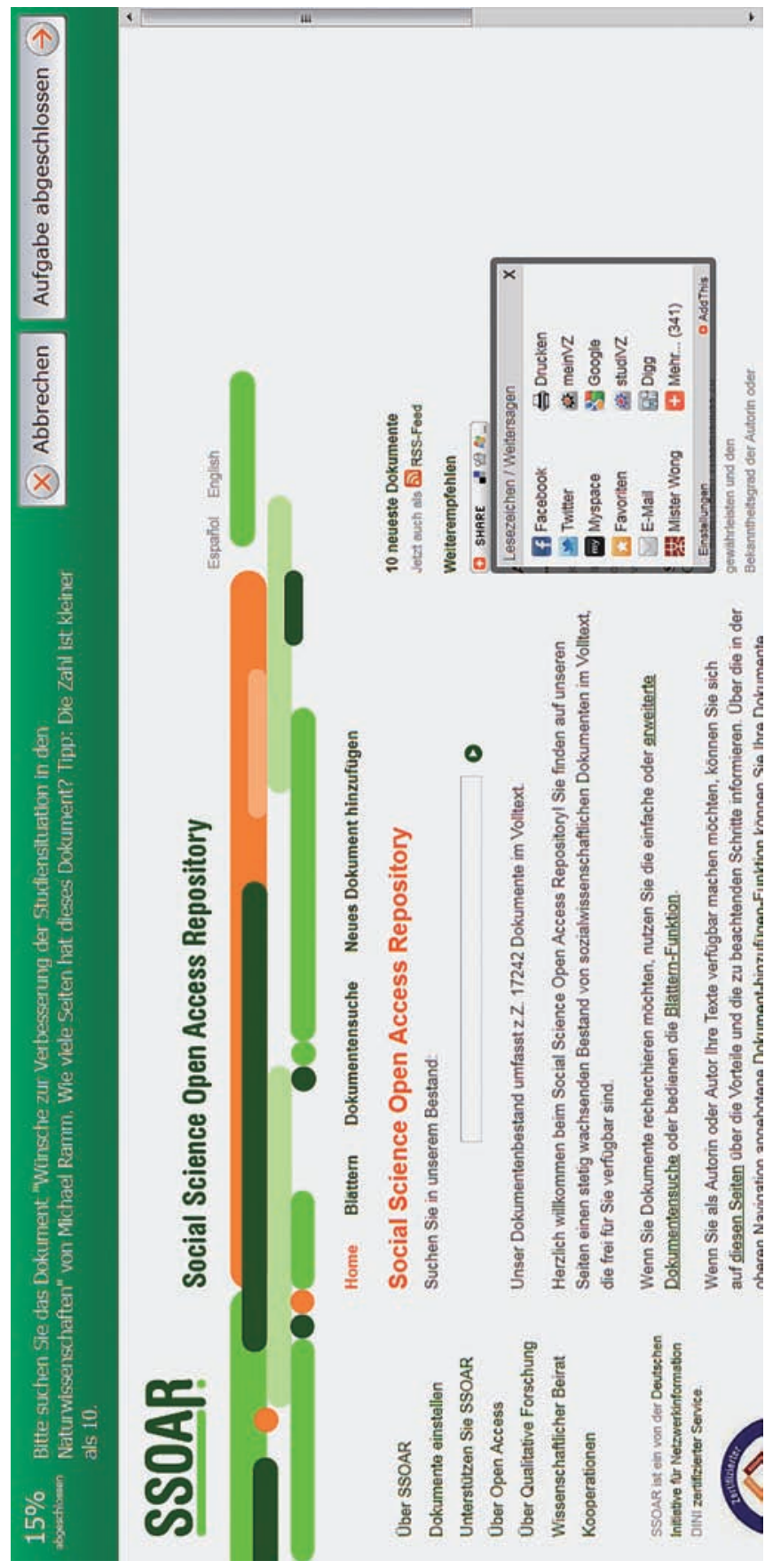

Abb. 4: Beispiel eines asynchronen Remote-Usability-Tests mit der Software Loop11 
möglicherweise potentielle Teilnehmer ausschließt, sowie die starke Abhängigkeit von der funktionierenden Technik auf beiden Seiten. Der Forscher arbeitet zudem unter verschärften rechtlichen und ethischen Bedingungen: Bei einem synchronen Test mit Bildschirmfreigabe erhält er Vollzugriff auf einen fremden Rechner und kann dort die Maus steuern. Eingaben wie Login-Daten können mitverfolgt und ausgelesen werden. Es wird angeraten, vor dem Test per Email ausführliche Informationen zu den Rechten des Teilnehmers und dem verantwortlichen Handeln des Forschers zu verschicken.

Asynchrone Remote-Usability-Tests sind orts- und zusätzlich zeitunabhängig. Je nach Anbieter sind die Tools zudem unabhängig vom Endgerät des Benutzers und können somit auch auf mobilen Geräten wie Smartphones Anwendung finden. Bei einem asynchronen Remote-Usability-Test greifen Benutzer auf das gewünschte Online-Angebot zu und bearbeiten dort wie bei einem Think-AloudTest konkrete Aufgaben. Diese erhält der Studienteilnehmer in Textform neben, über oder unter der angezeigten Website (Abb. 4).

Nach Erledigung der Aufgabe klickt der Teilnehmer - je nach System zum Beispiel auf „Aufgabe abgeschlossen“ bzw. „Aufgabe abbrechen“ oder gibt die Antwort in ein freies Feld ein oder wählt aus mehreren Antwortmöglichkeiten. Der Forscher kann zusätzlich durch Tracking den Weg des Benutzers nachverfolgen, sofern das getestete Angebot dies zulässt und es sich um permanente Links handelt. Zusätzlich zu den Aufgaben können durch Fragebogen-Elemente zum Beispiel die üblichen Daten wie demographische Angaben erfragt werden.

Im Gegensatz zu Usability-Tests mit kleinem Teilnehmerkreis in Laboren und daraus resultierenden qualitativen Daten, haben solche Tests vorwiegend quantitativen Charakter. Man erfährt, wie viele Teilnehmer eine Aufgabe lösen konnten und wie viele abbrachen und man lernt, wie viel Zeit Teilnehmer für eine bestimmte Aufgabe benötigten und an welchen Stellen die meisten Teilnehmer aufgaben. Zusätzlich kann man bei asynchronen Remote-Usability-Tests mehrere digitale Angebote miteinander vergleichen.

\section{Online-Interviews}

Die drei folgenden Methoden sind von qualitativer Natur; sie benutzen extern verfügbare Werkzeuge, um mit Benutzern im Internet in Kontakt zu treten. Dies sind die Online-Interviews, die Online-Fokusgruppen und die OnlineBeobachtungen. $\mathrm{Da}$ in der Online-Forschung über viele Jahre die zusätzlich benötigten Werkzeuge nicht verfügbar waren, dominierten Web-Fragebogen und Logfile-Analysen. Denn die Schwierigkeit qualitativer Online-Forschung ist leicht ersichtlich: Wie führt man im Internet mit jemandem ein Interview, der weder im gleichen Raum noch in derselben Zeitzone ist? Der Teilnehmer eines Online-Interviews muss neben der Software für VoiP (Voice over IP) 
auch die nötige Hardware wie Kopfhörer und Mikrofon besitzen. Viele OnlineForscher greifen daher immer noch auf die bewährte Methode des analogen Telefon-Interviews zurück.

Neben unterschiedlichen Zeitzonen erschweren verschiedene Sprachen der Benutzer die Durchführung von Online-Interviews. Bei einem internationalen Angebot wie zum Beispiel der Europäischen Digitalen Bibliothek Europeana.eu kann man entweder alle Interviews in Englisch führen oder aber möglichst viele Sprachen zur Verfügung stellen. Das Interview in Englisch hat den Nachteil, dass im ungünstigsten Fall weder der Interviewer noch der Teilnehmer Englisch als Muttersprache sprechen und es leicht zu Verständnisschwierigkeiten kommen kann. Schwieriger als bei Web-Fragebogen ist auch die Rekrutierung von Teilnehmern. Man kann auf einem Online-Angebot einer Einrichtung einen Link zur Studie setzen, über den sich Teilnehmer für das Interview bewerben können. Teilnehmer und Interviewer vereinbaren dann gemeinsam einen Termin und klären die technischen Anforderungen. Eine zweite Variante ist die direkte Aufforderung, in ein Interview zu treten, sobald ein Benutzer auf das Angebot klickt. Dies funktioniert ähnlich wie ein Pop-Up.

Zwei Nachteile ergeben sich aus letztgenannter Variante: Erstens erreicht man nur diejenigen Benutzer, die zufällig zu einer bestimmten Zeit online sind. Da man nachts sicherlich keine Interviews führen will, wird man bestimmte Benutzer niemals erreichen. Man hat also ein Problem mit der Stichprobe. Zweitens erfordern qualitative Interviews sehr viel Zeit: 30 Minuten Interviewzeit ist sehr kurz, eine Stunde die Regel und 90 Minuten die maximale Obergrenze. Die wenigsten Teilnehmer werden jedoch spontan 60 Minuten für ein Interview Zeit haben. Bei einem qualitativen Interview ist es deshalb wichtig, eine angemessene Aufwandsentschädigung zu bezahlen.

Es gibt verschiedene Werkzeuge, um online Interviews durchzuführen. Die gängigste Variante ist das Chat-Interview. Hierbei tippt man die Fragen ein und die Teilnehmer geben sofort eine Antwort. Der Vorteil ist, dass man das Interviewmaterial nicht mehr abtippen muss, da es bereits als Text vorliegt. Die Textgebundenheit wirkt sich jedoch auch nachteilig für die Inhaltsdichte aus, da Teilnehmer generell mündlich mehr erzählen als sie aufschreiben. Eine Alternative sind asynchrone E-Mail-Interviews, bei denen es eine Zeitverzögerung zwischen Frage und Antwort gibt. Vielfach verliert man jedoch Teilnehmer bei dieser Interviewform, da einige auf die zweite oder dritte Frage nicht mehr antworten. Eine Studie von $2009^{18}$ zeigt, dass E-Mail-Antworten in der Regel kürzer sind als im Chat; inhaltlich gibt es jedoch keine Differenz zwischen beiden Interviewformen.

18 Hussain \& Griffiths, 2009. 


\section{Online-Fokusgruppen}

Online-Fokusgruppen funktionieren ähnlich wie Online-Interviews, nur dass mehrere Teilnehmer anwesend sind und das Ziel der Studie nicht dasselbe ist. Untersucht man bei einem Online-Interview die Meinungen und das Verhalten einer Person, so interessiert man sich bei der Online-Fokusgruppe für Ergebnisse, die eine Gruppe hervorbringt. Ähnlich schwierige Bedingungen wie bei den Online-Interviews existieren bei den Werkzeugen für Online-Fokusgruppen. Die einfachste und bis jetzt immer noch gängigste Variante sind asynchrone Online-Fokusgruppen über Foren. Man eröffnet eine Diskussion mit einer Frage. Die Teilnehmer können nun antworten oder andere Antworten kommentieren. Die Vorteile dieser Form sind offensichtlich: Ein Forum ist einfach aufzusetzen und jeder kann zeit- und ortsunabhängig daran teilnehmen. Teilnehmer können gegebenenfalls sogar in ihrer eigenen Sprache antworten. Der Nachteil dieser Form: Fokusgruppen sind für den Moderator ohnehin nicht leicht zu steuern; in einem Forum ist es aber noch schwieriger, die stillen Teilnehmer zur aktiven Teilnahme zu überreden, zumal alles einmal Gesagte nachlesbar bleibt. Eine nicht ganz durchdachte Idee, die jemand mündlich äußert, ist schnell vergessen. Dieselbe Idee verschriftlicht ist für alle bis zum Ende der Fokusgruppe sichtbar, was manche Teilnehmer hemmt. Ein guter Moderator kann sich aber darauf vorbereiten und durch Regeln des guten Miteinanders innerhalb der Fokusgruppe vorbeugen.

Online-Fokusgruppen können auch in sogenannten Multi User Dungeons (kurz MUD) oder in virtuellen Welten wie Second Life stattfinden. Vielleicht die Form der Zukunft sind kleine virtuelle Konferenzräume, die man bucht und in die sich jeder Teilnehmer ohne Installation zusätzlicher Software eintragen kann. Benötigt werden auch hier nur ein Mikrofon und ein Kopfhörer, von Vorteil ist eine Webcam. Die bekannten privaten Chaträume, in die man sich aus einem öffentlichen Chat zurückziehen kann, standen Modell für die nun existierenden virtuellen Konferenzräume. Momentan gibt es bereits sehr viele Angebote, die jedoch nicht alle in der Ton- und Videoqualität überzeugen.

Ein weiterer Vorteil dieser virtuellen Konferenzräume sind integrierte virtuelle Whiteboards, auf die Teilnehmer selbst zeichnen und schreiben können oder auf denen sie ein existierendes Angebot kommentieren können (Abb. 5). Mittels dieser Whiteboards kann man auch Studien mit dem Card-Sorting-Test durchführen. Bei dieser Methode überprüfen die Teilnehmer den Aufbau eines digitalen Angebots. Auf gelben Karten befinden sich zum Beispiel die Überschriften oder Namen der jeweiligen Unterseite eines Online-Angebots. Auf grünen Karten befinden sich dann einzeln aufgelistet alle Elemente, die sich unter diesen Überschriften befinden. Der Studienteilnehmer muss dann die grünen Karten den gelben Karten zuordnen; im Anschluss wird überprüft, ob die Vorstellung der Benutzer mit den Zuordnungen der Einrichtung übereinstimmt. 


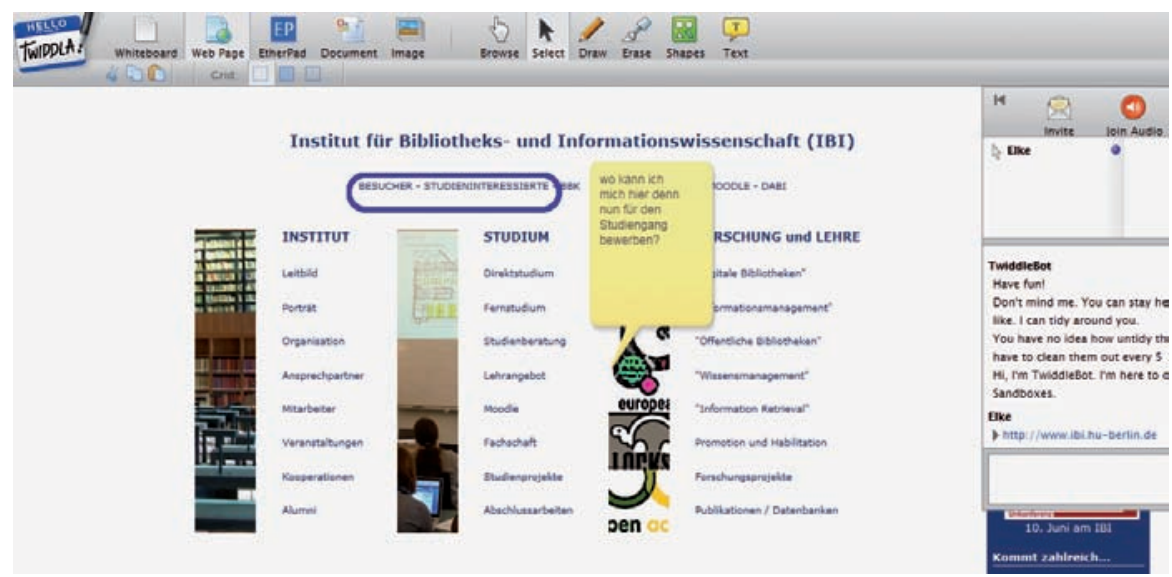

Abb. 5: Beispiel eines virtuellen Whiteboards (Softwareanbieter Twiddla.com)

\section{Qualitative Online-Beobachtungen}

Als letzte Methode gibt es verschiedene Formen der ethnografischen Herangehensweise. Die qualitative Beobachtung (engl. virtual observation) ähnelt im Internet der Inhaltsanalyse, da vorwiegend Texte als Untersuchungsobjekt verwendet werden. Beobachtet wird zum Beispiel, in welcher Form Reaktionen auf eine Meldung erfolgen. So untersuchten qualitative Forscher im Internet die Verbreitung und Bewertung der Meldung, dass Michael Jackson gestorben ist. Methodisch kann man aktiv oder passiv am Online-Geschehen teilnehmen und sich Beobachtungen über die Form der Interaktion notieren. Erlaubt eine digitale Bibliothek die aktive Mitarbeit von Benutzern wie die Vergabe von Tags oder von Benutzern verfasste Rezensionen oder Kommentaren, dann kann man diese qualitativ untersuchen. Dies trifft auch auf digitale Angebote zu, die zum größten Teil von Benutzern gefüllt werden. Die soziale Plattform FlickR oder das Bildarchiv Prometheus gehören dazu. Untersucht werden könnte dann zum Beispiel welche Inhalte von wem zu welchem Zweck verfügbar gemacht werden. Auf Basis dieses Wissens kann man die eigenen Angebote benutzerorientiert gestalten.

Bei einer Online-Beobachtung an der North Carolina State University entwickelte man eine Anwendung für den iPad und andere Tablet-PCs, mit denen man mobil Daten erfassen kann. ${ }^{19}$ Ziel der Studie war es, mehr über die Benutzung der Bibliotheksräume herauszufinden, insbesondere wie sich OnlineNutzung und reale Raumnutzung verknüpfen.

19 Casden, 2011. 


\section{Rechtliche Einschränkungen bei Online-Studien}

Neben vielen Vorteilen birgt die Online-Forschung einige Tücken, auf die der folgende Teil dieses Beitrags eingeht. Es handelt sich dabei um technische Tücken, um rechtliche Einschränkungen und um Fragen bezüglich der Validität von Online-Studien.

Auf rechtlicher Ebene gibt es einige Vorgaben, die auch auf die OnlineForschung zutreffen. Beispielhaft sei hier auf die Richtlinie 95/46/EG des Europäischen Parlaments und des Rates vom 24. Oktober 1995 zum Schutz natürlicher Personen bei der Verarbeitung personenbezogener Daten und zum freien Datenverkehr (auch bekannt als European Union Data Protection Directive) hingewiesen. ${ }^{20}$ Diese besagt, dass Studienteilnehmer oder - allgemeiner Untersuchungssubjekte (wie zum Beispiel Verfasser von Weblogbeiträgen):

- explizit darüber informiert werden müssen, welche personengebundenen Daten über sie gesammelt werden,

- informiert werden müssen, warum persönliche Daten überhaupt gesammelt werden,

- in der Lage sein müssen, fehlerhafte Daten zu korrigieren,

- in der Lage sein müssen, aus der Datensammlung auszusteigen („,Opt-out“),

- davor geschützt werden müssen, dass ihre personengebundenen Daten in ein anderes Land transferiert werden, in dem es weniger stringente Regelungen zur Privatsphäre gibt ${ }^{21}$.

\section{Technische Tücken bei der Online-Forschung}

Wichtigster Baustein der Online-Forschung ist die Nutzung technischer Hilfsmittel. Dies kann das Werkzeug für den Web-Fragebogen sein, die Software, um Logfiles aufzuzeichnen oder aber auch die Kamera und das Mikrofon, um Online-Interviews durchzuführen. Aber die starke Abhängigkeit von der Technik birgt Gefahr: wenn bei der Testdurchführung die Technik versagt, bleibt nur der Abbruch der Studie.

Einige potentielle Probleme können bereits vor Studienbeginn vermieden werden. So muss die eigene Studie nicht nur auf den Inhalt, sondern auch auf das technische Funktionieren getestet werden. Studien sollten unter verschiedenen Computer-Betriebssystemen und mit verschiedenen Browsern getestet werden. Nicht jeder Fragebogen funktioniert mit einem Opera-Browser und nicht jeder Remote-Usability-Test funktioniert auf mobilen Endgeräten.

20 Vgl. Schutz von personenbezogenen Daten 1995.

21 Vgl. Ess, 2002. 
Es ist wichtig, die betroffenen Kollegen frühzeitig über die Studie zu informieren, damit nicht am ersten Tag der Durchführung der Server gewartet wird oder ein Relaunch der zu testenden Website ansteht. Das Funktionieren der Technik auf Seiten der Teilnehmer ist schwieriger zu kontrollieren. Im Grunde kann man nur durch eine gute Kommunikation die gröbsten Probleme verhindern. So ist es wichtig, dass man die Teilnehmer der Studie darüber informiert, welche technischen Anforderungen an ihre Ausrüstung gestellt werden: wird ein Mikrofon oder eine Kamera benötigt und reicht die Geschwindigkeit der Internetübertragung für ein Online-Interview? Benötigen die Teilnehmer Administratorenrechte, um zum Beispiel eine Software für einen synchronen Remote-Test zu installieren? Oder ist es für einen bestimmten Test notwendig, dass der Remote-Rechner Cookies setzen darf oder dass Javascript ausgeführt werden kann?

So verheißungsvoll Remote-Usability-Tests für die Benutzerforschung sind, so bergen sie gleich ein dreifaches technisches Risiko: erstens kann das zu testende Online-Angebot auf Seiten des Forschenden versagen. Zweitens kann die Technik, wie eben beschrieben, auch beim Teilnehmer Probleme verursachen. Und drittens könnte die Technik auf Seiten des Anbieters der Usability-Software nicht funktionieren. So kann es zum Beispiel passieren, dass der Server, auf dem der Test läuft, in Nordamerika liegt und dort nachts Serverarbeiten durchgeführt werden, während Studienteilnehmer hierzulande den Test aufrufen wollen. Hier ist eine genaue Abstimmung mit dem Softwareanbieter hilfreich. Ein gut gepflegter Support ist gerade bei Remote-Usability-Software sehr wichtig.

Da man nicht jede technische Tücke durch Pretests und gute Kommunikation verhindern kann, sollte man bei jeder Studie von Beginn an einen ausreichenden zeitlichen und personellen Puffer einplanen. Dann ist es weniger gravierend, wenn eine Studie sich um ein paar Tage verzögert oder wenn man zusätzliche Teilnehmer benötigt.

Ein viel größeres Problem der Online-Forschung sind möglicherweise nicht die Tücken in der Benutzung der Technik, sondern das Fehlen der Technik an sich. Sollte jemand automatisch von einem synchronen Remote-Usability-Test oder einem Online-Interview ausgeschlossen werden, nur weil er kein Mikrofon besitzt? Das Thema Barrierefreiheit ist ein ernsthaftes Problem für die Validität der Online-Forschung, da von Beginn an bestimmte Nutzergruppen als Studienteilnehmer ausgeschlossen werden. Können Online-Studien repräsentativ sein, wenn man die Stichprobe auf Basis der Verfügbarkeit einer bestimmten Technik zieht und die Technik dabei nicht für die Benutzung des zu untersuchenden digitalen Angebots nötig ist? Auf diese Fragen gibt der folgende Abschnitt zur Validität von Online-Studien Antwort. 


\section{Validität von Online-Studien}

Eine repräsentative Studie erlaubt es, Aussagen über eine Grundgesamtheit zu treffen. Doch wie sieht die Grundgesamtheit einer digitalen Bibliothek aus? Sind das alle Internetuser oder nimmt man nur die Zahl der potentiellen Benutzer, die man von lokalen Erhebungen kennt, so wie der BIX sie für seine virtuelle Messung als „,primäre Nutzergruppe“22 definiert? Im Gegensatz zu einer physischen Bibliothek gibt es keine konkreten Angaben darüber, wer Teil der Grundgesamtheit aller Benutzer eines bestimmten Service ist (Angebote mit Anmeldung sind hiervon natürlich ausgeschlossen). Die Angabe, dass über 3.000 Benutzer an einem Fragebogen teilgenommen haben, sagt nichts über die Repräsentativität der Studie aus. Die 3.000 Benutzer könnten besonders aktive Benutzer oder besonders zufriedene Benutzer sein. Ohne ein detailliertes Wissen über die Grundgesamtheit kann auch keine ,repräsentative“ Stichprobe gezogen werden. Das ist das wohl größte Manko der Online-Forschung: Eine repräsentative Erhebung ist schlichtweg unmöglich.

Neben dem bereits angesprochenen Problem der Repräsentativität durch das Hindernis, dass der Teilnehmer eine bestimmte Technologie verfügbar haben muss, gibt es ein weiteres Problem: die Convenience Samples. Bittet man Benutzer auf einer Website oder in einer Mailingliste um die Teilnahme an einer Studie, so ziehen nicht die Forscher gezielt eine Stichprobe, sondern jeder Benutzer entscheidet selbst, ob ihn eine Teilnahme interessiert. Damit findet eine Selbstselektion der Teilnehmer statt, die häufig in einer Überproportionierung von besonders motivierten oder besonders angesprochenen Teilnehmern resultiert. Haben zum Beispiel einige Benutzer Probleme mit dem Interface, könnten diese viel eher motiviert sein, ein Feedback abzugeben, als Benutzer, die problemlos mit dem System interagieren.

Es gibt verschiedene Herangehensweisen, wie man diese Begrenztheiten der Online-Forschung abmildern kann. Um eine bessere Zufallsstichprobe zu erhalten, sollte man nicht pauschal jeden Benutzer, sondern zum Beispiel nur jeden n-ten Benutzer um die Teilnahme an der Studie bitten, damit zumindest eine Zufallsauswahl der gefragten Teilnehmer erfolgt. Technisch umsetzbar ist dies durch gezielt gesteuerte Pop-Ups. Alternativ kann man vor der eigentlichen Studie eine Vorerhebung schalten, in der man die Benutzer nach ihren Gewohnheiten und demographischen Angaben fragt und auf Basis dieses BenutzerPools dann eine geeignete Stichprobe auswählt. Bei dieser Variante bleibt der hohe Faktor der Selbstselektion erhalten, da Benutzer sich bereit erklären müssen, gleich zweimal an einer Studie teilzunehmen (die Vorabfrage und die eigentliche Studie).

Eine in den Sozialwissenschaften häufig verwendete Form ist die Benutzung von Panels. Panels sind eine Gruppe von potentiellen Teilnehmern, die sich

22 BIX Handbuch - WB 2011. 
bereit erklärt haben, regelmäßig an Studien teilzunehmen. Panelmitglieder werden aktiv rekrutiert, um nahe an die Gesamtpopulation heranzukommen. Panels müssen regelmäßig erneuert, aktualisiert und gepflegt werden. ${ }^{23}$ Dank der besseren Repräsentativität von Panels im Vergleich zu anderen Rekrutierungsmethoden, werden Panels - insbesondere solche, die Teilnehmer auch offline rekrutiert haben - gerne auch als Königsweg der Onlineforschung bezeichnet.

Zwei Elemente des Forschungsdesigns sind für die Online-Forschung von besonderer Bedeutung: Erstens ist es wichtig, die richtigen Variablen abzufragen. Welche Variablen bilden einen guten Indikator für das Nutzerverhalten? So ist es möglicherweise bedeutsam, die Anzahl an weiteren Applikationen zu erfassen, die während der Bearbeitung einer Studie geöffnet waren. ${ }^{24}$ Zweitens ist eine ausreichend lange Feldzeit bei Online-Studien wichtig, da eine Verkürzung der Feldzeit zu geringeren Teilnahmequoten und zu einer verzerrten Ausschöpfung führt. So nahmen nur „26 Prozent der zur Online-Befragung aufgeforderten Personen [...] sehr zeitnah (innerhalb 48 Stunden)" an einer Studie von Lanninger $^{25}$ teil. Im Durchschnitt nahmen deutlich mehr Männer in einem kürzeren Zeitraum als Frauen teil.

Wie bereits im Abschnitt zu Remote-Usability-Testing angedeutet, ist ein ausreichend bemessener Zeitplan für die Online-Forschung unerlässlich. In einer Studie von Stieger und Göritz ${ }^{26}$ nahmen in etwa die Hälfte der angefragten Benutzer letztendlich an einer Studie mit Instant-Messaging teil. Ähnliche Quoten gelten für das Remote-Usability-Testing. Auch ist die Gefahr eines Abbruchs während der Studie deutlich höher, als wenn Benutzer physisch an einer Studie teilnehmen. So berichten Stieger und Göritz von 10,5 Prozent der Teilnehmer, welche den Chat während der Studie verlassen haben.

\section{Zusammenfassung}

Die Methoden der Online-Forschung sind eine Bereicherung für die Forschung über digitale Bibliotheken. Sie eröffnen die Möglichkeit, nahe an den tatsächlichen Benutzern zu sein. Remote-Usability-Tests untersuchen digitale Angebote auf den Endnutzergeräten und nicht auf Laborrechnern. Aus Online-Forschung erhaltene Daten spiegeln die tatsächliche Nutzungssituation adäquater wider, als manche herkömmliche „Offline-Methode“ dies konnte.

Zum ersten Mal ist es möglich, Benutzer aus der ganzen Welt auch in qualitative Studien mit einzubeziehen, ohne gleichzeitig hohe Reisekosten zu generieren. Die rasante technische Entwicklung neuer Werkzeuge verspricht

23 Theobald, 2000.

24 Greifeneder, 2011.

25 Lanninger, 2001.

26 Stieger; Göritz, 2006. 
auch für die nächsten Jahre weitere Hilfsmittel, um Online-Forschung noch besser durchführen zu können.

Trotz aller Euphorie über Online-Forschung sollten die Beschränkungen derselben nicht vergessen werden. So sind die Werkzeuge der Online-Forschung zugleich Segen und Fluch. Technische Hilfsmittel wie virtuelle Whiteboards ermöglichen ganz neue Ansätze in der Forschung zu digitalen Bibliotheken. Aber sie schließen Teilnehmer ohne die nötigen Zugangsmittel aus. Convenience Samples werden noch für viele Jahre die Qualität der Online-Forschung schmälern.

Gute Online-Forschung zu digitalen Bibliotheken hängt davon ab, dass man sich der genannten Beschränkungen deutlich bewusst ist und die erhaltenen Daten unter Einbeziehung dieser Beschränkungen interpretiert. Denn erst dann bieten die Online-Werkzeuge wirklich einen Mehrwert für die Forschung zu digitalen Bibliotheken und nicht nur ein hübsches Beiwerk, das ein schlechtes Forschungsdesign kaschiert.

\section{Literaturverzeichnis}

Akselbo, Jeppe Lomholt u.a. „The hybrid library: from the users' perspective. A report for the DEFF project ,The loaners" expectations and demands for the hybrid library“". Edited by Denmark's Elektronic Research Library. http://www.statsbiblioteket.dk/summa/ fieldstudies.pdf (14.04.2010).

beluga. „Ein Katalog 2.0-Projekt der Hamburger Bibliotheken.“ http://beluga-blog.sub.unihamburg.de/blog/ (17.06.2011).

Beschnitt, Martin (2009). ,Standardisierte Fragebögen zur Messung der Usability/User Experience - derzeit wieder in Mode?" http://www.usabilityblog.de/2009/09/standardisiertefragebogen-zur-messung-der-usabilityuser-experience-derzeit-wieder-in-mode/ (01.06. 2011).

BIX Handbuch - WB. (2011). „Erhebungsunterlage für den BIX für wissenschaftliche Bibliotheken 2011 (Berichtsjahr 2010).“With assistance of Ulla Wimmer. http://www.bixbibliotheksindex.de/ (16.06.2011).

Buchanan, Elizabeth A. (2004). „Readings in virtual research ethics. Issues and controversies.“ Hershey, Pennsylvania: Information Science Publishing.

Casden, Jason and Chapman Joyce. (2001). „Building a staff-facing tablet application for library assessment. Code4lib.“ North Carolina State University Libraries, 2011. http:// www.slideshare.net/casden/building-an-open-source-stafffacing-tablet-app-for-libraryassessment (01.06.2011).

Clickheat. http://www.labsmedia.com/admin/clickheat/index.php (17.06.2011).

Ess, Charles. (2002). „Ethical decision-making and Internet research. Recommendations from the aoir ethics working committee." With assistance of AoIR ethics working committee, 2002. http://www.aoir.org/reports/ethics.pdf (24.05.2011).

Greifeneder, Elke. (2011). „The Impact of Distraction in Natural Environments on User Experience Research." Conference on Theorie and Practice of Digital Libraries.

Greifeneder, Elke; Axel Kuhn und Sandra Rühr. (2011). „Aufbau und Entwicklung einer digitalen Buchgeschichte. Studie zu Anforderungen und Zielgruppen.“ ALLES BUCH. 
Studien der Erlanger Buchwissenschaft. Hrsg. Von Ursula Rautenberg und Volker Titel. Universität Erlangen - Nürnberg (XXXIV), S. 4-27. http://www.alles-buch.uni-erlangen. de/34_DigiBuch_neu.pdf (17.06.2011).

Hine, Christin. (2006). „Virtual methods. Issues in social research on the Internet.“ Paperback ed. reprinted. Oxford: Berg.

Hussain, Zaheer and Mark D. Griffiths. (2009). „The Attitudes, Feelings, and Experiences of Online Gamers. A Qualitative Analysis.“ Cyberpsychologie \& Behavior, H. 12 S. 747753. DOI: $10.1089=$ cpb.2009.0059 (14.05.2010)

Krug, Steve und Jürgen Dubau. (2006). „Don’t make me think! Web Usability - das intuitive Web.“ 2. Aufl. Bonn: mitp.

Kuniavsky, Mike. (2003). „Observing the user experience. A practitioner's guide to user research." San Francisco, California: Morgan Kaufmann (The Morgan Kaufmann series in interactive technologies).

Lanninger, Thomas. (2001). „Methodentests als Basisvoraussetzung für Online-Forschung. Stichprobeneffekte im Split-Half-Design Cati - Online." planung \& analyse (2001) H. 6 S. 50-54.

Markham, Annette N. and Nancy K. Baym (eds.) (2009). „Internet inquiry. Conversations about method." Los Angeles: Sage Publications.

Nielsen, Jakob. (2000). „Designing Web Usability.“ Indianapolis Indiana: New Riders.

Remote \& Online Usability Testing Tool | Loop11. http://www.loop11.com/ (21.10.2010).

Schutz von personenbezogenen Daten. (1995). „Richtlinie 95/46/EG des Europäischen Parlaments und des Rates vom 24. Oktober 1995 zum Schutz natürlicher Personen bei der Verarbeitung personenbezogener Daten und zum freien Datenverkehr.) Amtsblatt L 281 vom 23.11.1995. http://europa.eu/legislation_summaries/information_society/114012_de. htm (17.06.2011)

Shneiderman, Ben and Catherine Plaisant. (2005). „Designing the user interface. Strategies for effective human-computer interaction.“ 2. Aufl. Boston: Addison - Wesley.

Stieger, Stefan und Anja Göritz. (2006). „Using Instant Messaging for Internet-Based Interviews." Cyberpsychologie \& Behavior 9 (2006) H. 5 S. 552-559. http://wisopsychologie. uni-erlangen.de/mitarbeiter/goeritz/Cyberpsych.pdf (14.05.2010).

Taylor, R. S. (1991). „Information use environments.“ Progress in communication sciences. Edited by Dervin, Brenda J. and Melvin J. Voigt. Norwood, New Jersey: Ablex Publishing 1991. S. 217-255.

Theobald, Axel. (2000). „Determinanten des Online Research.“ planung \& analyse (2000) H. 5 S. $72-76$.

Troll Covey, Denise (2002). „Usage and usability assessment. library practices and concerns." Edited by the Digital Library Federation. Council on Library and Information Resources. Washington, DC. http://www.diglib.org/pubs/dlf096/dlf096.htm (05.02. 2009).

twiddla.com. http://www.twiddla.com/ (08.03.2011).

Welker, Martin und Olaf Wenzel. (2007). „Online-Forschung 2007. Grundlagen und Fallstudien.“ Köln: von Halem (Neue Schriften zur Online-Forschung, 1).

Winter, Stefanie. (2000). Quantitative vs. Qualitative Methoden. http://imihome.imi.unikarlsruhe.de/nquantitative_vs_qualitative_methoden_b.html 